\title{
Digital Radio over Fibre for Future Broadband Wireless Access Network Solution
}

\author{
S. R. Abdollahi, H.S. Al-Raweshidy \\ WNCC Group, School of Eng. and Design, \\ Brunel University, \\ Uxbridge, Middlesex, UB8 3PH, UK. \\ E-mail: \{seyedreza.abdollahi, hamed al-raweshidy, \\ rajagopal.nilavalan\}@brunel.ac.uk
}

\author{
S. Mehdi Fakhraie*, and R. Nilavalan \\ *ECE Department, College of Engineering, \\ University of Tehran, \\ North Kargar Ave., Tehran, 14395-515, I. R. of \\ Iran. \\ E-mail: fakhraie@ut.ac.ir
}

\begin{abstract}
Digital systems are more flexible and environment-process-tolerant than analogue systems. They are more reliable and robust against cross-talk, interference and channel noises, and are capable of covering higher dynamic range than analogue systems. Wideband electronic analogue to digital conversion (ADC) systems have critical problems encountered in highfrequency broadband communication systems that the recent electronic ADCs (EADC) have experienced those such as uncertainty of sampling time. In this paper, an 80Gigasample/s all photonic sampling and quantization ADC and photonic digital to analogue conversion system with six effective number of bits (ENOB) is designed. By using this Photonic ADC (PADC), a digital radio over fibre link for wireless radio frequency (RF) signal transportation over $50 \mathrm{~km}$ single mode fibre has been designed whose performance is investigated in this paper.
\end{abstract}

Keywords- PADC; Radio over Fibre; ENOB.

\section{INTRODUCTION}

Radio over fibre technology is currently receiving large attention due to its ability to provide simple antenna front ends, increased capacity, and wireless access coverage. Radio over Fibre system (RoF) is the technique of modulating the radio frequency (RF) subcarrier onto an optical carrier for distribution over a fibre network. RoF technique has been considered a costeffective and reliable solution for the distribution of the future wireless access networks by using optical fibre with vast transmission bandwidth capacity. RoF link is used in remote antenna applications to distribute signals for Microcell or Picocell base station [1][2][3]. The downlink RF signals are distributed from a central station (CS) to many base station (BS) known as Radio Access Point (RAP) through the fibres. The uplink signals received at RAP are sent back to the CS for any signal processing. RoF has the following main features: (1) it is transparent to bandwidth or modulation techniques. (2) Needs simple and small BSs. (3) Centralized operation is possible. New wireless subscribers are signing up with an increasing demand of more capacity for ultra-high data rate transfer at speeds of $1 \mathrm{Gbp} / \mathrm{s}$ and up, while the radio spectrum is limited. This requirement of more bandwidth allocation places heavy burden on the current operating radio spectrum and causes spectral congestion at lower microwave frequency. Millimetre Wave (MM-Wave) communication system offers a unique way to resolve these problems [4].

Digital signal processing has revolutionized modern communication systems by offering unprecedented performance and adaptivity. Since digital systems are flexible and more conveniently interface with other systems, and are more reliable and robust against additive noises of devices and channel and achieve better dynamic range than analogue systems. Analogue to digital and digital to analogue converters (ADC and DAC, respectively) are the link between the analogue world and the digital world of signal processing and data handling. In an analogue system the bandwidth is limited by devices performance and parasitic components introduced. Thermal noise generated in active and passive components limits the dynamic range of an analogue system. The ratio between the maximum allowable analogue signal and the noise level determines the dynamic range of the system.

Wideband analogue to digital conversion is a critical problem encountered in broadband communication and radar systems. The recent electronic analogue to digital conversion systems experience problems such as jitter in sampling clock, settling time of the sample and hold circuit, speed of comparator, mismatches in the transistor thresholds and passive component values. These limitations imposed by all of these factors become more severe at higher frequencies. PADCs by using the Mode-locked laser (MLL) and Mach Zehnder Modulator (MZM) are able to scale the timing jitter of the laser sources to the sub-femtosecond level, which will allow the designers to push the resolution bandwidth by many orders of magnitude beyond what electronic sampling systems can achieve currently [5].

This paper is organized as follows: Section II describes analogue RoF (ARoF) and Section III introduces the digital RoF (DRoF) system and its architecture. Section IV provides our system simulation results. Finally, conclusions are presented in Section V.

\section{AnAlogue RoF System}

An analogue RoF ( ARoF) link that includes optical source, modulator, optical amplifier \& filters, optical channel and photodiode as a receiver and electronic amplifiers and filters. In some cases the optical source is 
directly modulated by RF signal, but as the laser is usually a significant source of noise and distortion in a radio over fibre link, so laser diode normally exhibits nonlinear behaviour. When it is well driven above its threshold current, its input/output relationship can be modelled by Volterra series of order 3 [6]. So, in the recent RoF systems, semiconductor laser is used for the optical source and external modulator. Therefore, analogue optical link suffer from nonlinearity of both microwave and optical components that constitute the optical link.

Usually a Mach Zehnder interferometer fabricated from LiNbO3, impress the RF signal on the optical intensity. Also, a wide range of other modulator can be used [7][8].

\section{DigITAL RoF SYSTEM}

The digital RoF (DRoF) link can maintain the dynamic range more independent than optical fibre link distance and can employ the present infrastructure for transporting the digitized radio traffic [1][9]. Fig. 1 shows the proposed digital radio over fibre system. In this system, the analogue RF signal has been digitized by using the PADC. With generation of digital data and proportional multiplexing technique, data stream is transported over optical fibre network. In this design, both the baseband and digitized RF data traffic signals are transported through metro and access networks by using wavelength division multiplexing technique. For compensating the chromatic dispersion, the system uses the chromatic dispersion compensation fibre.

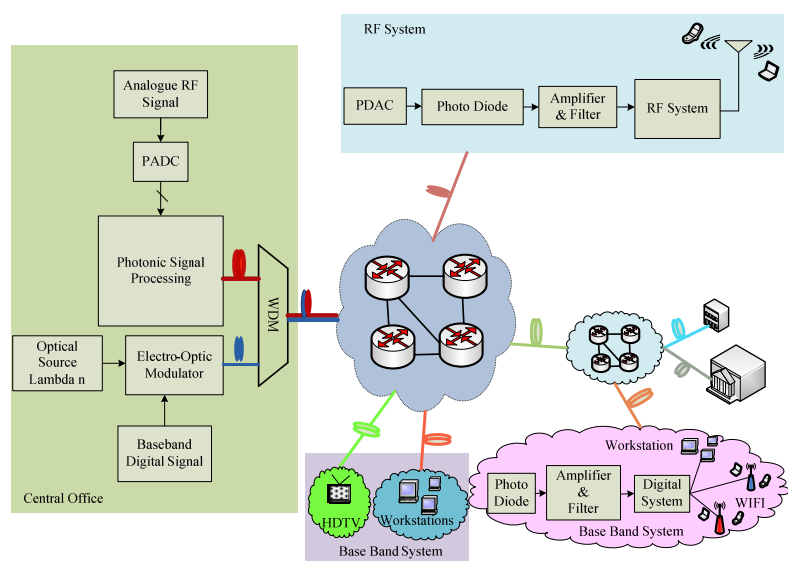

Figure 1: The proposed downlink DRoF system.

The photonic DAC of RF system converts digital photonic signal to its analogue version of optical modulated signal. The RF signal is detected by single high speed photo diodes. The RF signal after processing by an electronic RF signal processing system has been fed to the base station transmitter antenna.

\section{A. Photonic Analogue-to-Digital Conversion}

High-speed sampling and quantization of photonic analogue-to- digital conversion (ADCs) systems have wide variety of applications in todays high-speed signal processing electronic circuits and communication systems [5][9][10]. Fig. 2 shows the proposed photonic sampling and quantization ADC system architecture.

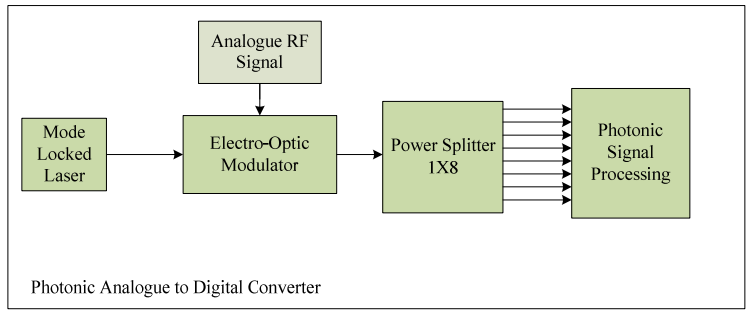

Figure 2: The photonic ADC’s architecture.

The digitized optical signal's power have been split into 8 channels and fed to photonic signal sampling and quantization block. In this block, the digitized signals have been processed, multiplexed and combined together for transporting over single mode fibre medium. In this architecture, eight-bit PADC has been designed. For performance investigation of PADC, the photonic quantized signals have been converted to electrical signals by using a high-speed photo diode array.

Despite the variety in ADC's, their performance can be summarized by a relatively small number of parameters, first: stated resolution (number of bits per sample), second: signal to noise ratio (SNR), third: spurious-free dynamic range (SFDR) and forth: power dissipation. SNR and SFDR are the most important parameters of dynamic performance for high-speed applications. The SNR and SFDR provide a more accurate measure of ADC performance than the stated number of bits. The SFDR is the ratio of the single-tone signal amplitude to the largest non-signal component within the spectrum of interest. The noise spectrum contains contributions from all the error mechanisms present. These include the noises of quantization, circuit, aperture, and aperture uncertainty and comparator ambiguity. The only error mechanism present in an ideal ADC is quantization [11].

The $\mathrm{Q}$ denoted as the quantization error is the difference between the analogue signal and digital sampled signal, which is the least significant bit (LSB) of the binary representation of that value given by Equation 1.

$$
\mathrm{Q}=\frac{\mathrm{V}_{\mathrm{FS}}}{2^{\mathrm{N}}}
$$

That value of $\mathrm{N}$ shows the ADC resolution bits and VFS is the full scale voltage. In this design $\mathrm{N}=8$ bits and VFS $=1$ Volt. Therefore, Q is $125 \mathrm{mV}$. To simplify the calculations, the error of quantization can be defined as a simple linear function during a sampling interval (T). Equation 2 denotes the quantization error function and the noise rms power given by Equation 3 .

$$
\mathrm{e}(\mathrm{t})=\mathrm{Q} \cdot\left(\frac{\mathrm{t}}{\mathrm{T}}-\frac{1}{2}\right)
$$




$$
\mathrm{NP}_{0}(\mathrm{rms})=\sqrt{\frac{\int_{0}^{\mathrm{T}}[\mathrm{e}(\mathrm{t})]^{2} \mathrm{dt}}{\mathrm{T}}}=\frac{\mathrm{Q}}{12}
$$

and the SNR calculation is given by Equation 4 .

$$
\begin{aligned}
\operatorname{SNR}(\mathrm{dB})= & 20 \log _{10}\left(\frac{\mathrm{V}_{\mathrm{FS}}(\mathrm{rms})}{\mathrm{NP}_{0}(\mathrm{rms})}\right) \\
= & 6.02 . \mathrm{N}+1.76 \mathrm{~dB}
\end{aligned}
$$

So, the SNR can be improved by increasing N. The effective number of bits is given by Equation 5:

$$
\mathrm{ENOB}=\operatorname{SFDR}(\mathrm{dBc}) / 6.02
$$

\section{B. Base Station}

Fig. 3 shows the block diagram of the down-link section of photonic and RF system at the base-station for the proposed DRoF architecture. In this system, the photonic DAC converts photonic digital waveform to analogue optical signal. By using the proposed PDAC, the necessity of optical to electrical conversion will be fulfilled by only a high-speed photo diode. For reducing the cost and complexity of the system, the optical sampling pulse has been reused at base-station for sampling the uplink RF signal. Therefore, the need for new optical carrier source and sampling pulse has been satisfied. In the uplink data transportation, the same technique has been implemented.

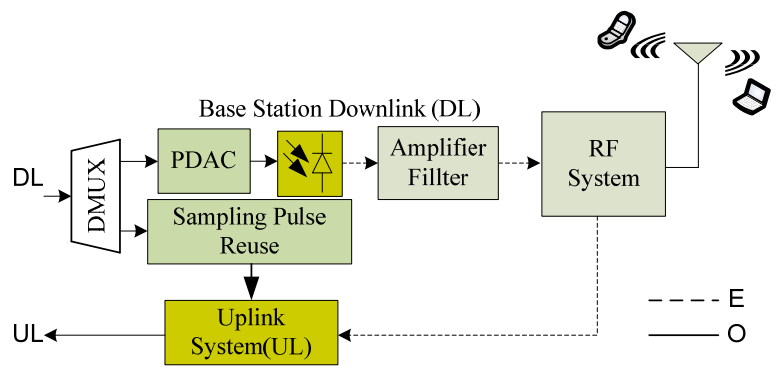

Figure 3: The Base-Station System that uses PDAC.

By realizing this scheme, it is possible to use free spectrum capacity of metro and access networks for transporting the broadband wireless and wireline data traffic. This technique centralises the signal processing, system management, and monitoring processes. Therefore, wireless network could be integrated with existing optical networks that reduces the future superbroadband access-network-system implementation overheads and service costs to the end-users.

\section{Simulation Results}

In this section, the results of simulations that have been performed by Optiwave-Optisystem and Matlab environments are presented. According to the calculations and simulations the SNR of quantized signals are equal to $49.92 \mathrm{dBm}$. By noticing the simulation results that are shown in Fig. 4 the SFDR and ENOB at 160 Gigasample/s sampling rate are equal to $9.82 \mathrm{~dB}$ and 1.63, respectively.

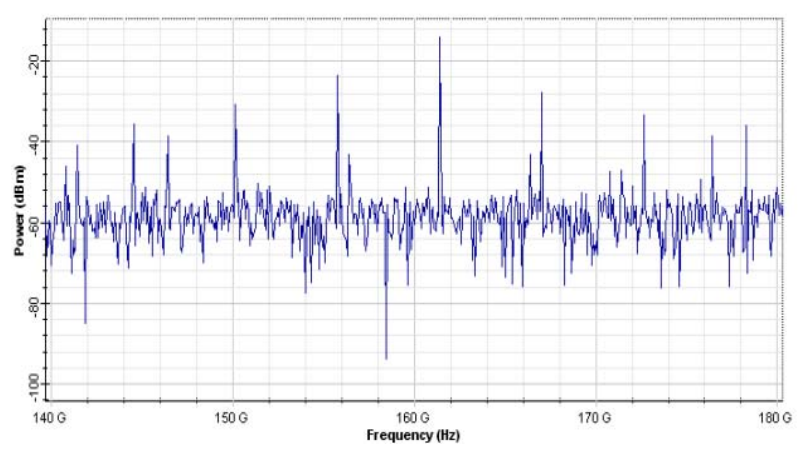

Figure 4: Power spectrum of the digital electrical signal.

For selecting the optimum sampling rate, the PADC performance has been investigated for various sampling rates. Fig. 5 shows the ENOB variations with sampling rate. The best ENOB resolution is evaluated at 80 Gigasample/s about 6.

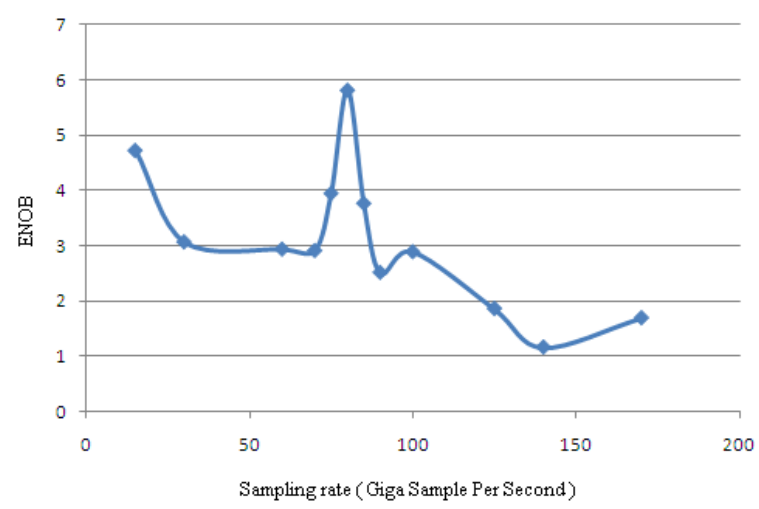

Figure 5: ENOB variation versus sampling rate.

As the transfer function of MZM is inherently nonlinear. The dynamic range has been converted into ENOB and plotted against the RF link sampling rates. The simulations show that using a nonlinear modulator severely limits the PADC resolution for practical applications.

Fig. 6 shows a random sample sequence Quadrature Amplitude Modulated (QAM), RF signal, the sampled signal, and two MSB and LSB quantized digital optical signals. In this design, sampling and quantization have been implemented in optical domain. As illustrated in the figure, the $80 \mathrm{GHz}$ optical sampling pulse is generated by a $1550 \mathrm{~nm}$ mode-locked laser and a sample $10 \mathrm{GHz}, 64 \mathrm{QAM}$, RF signal has been fed to MZM electro-optical modulator as a photonic sampling device.

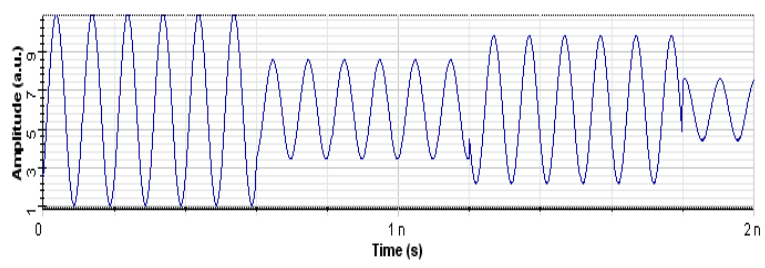

(a) 


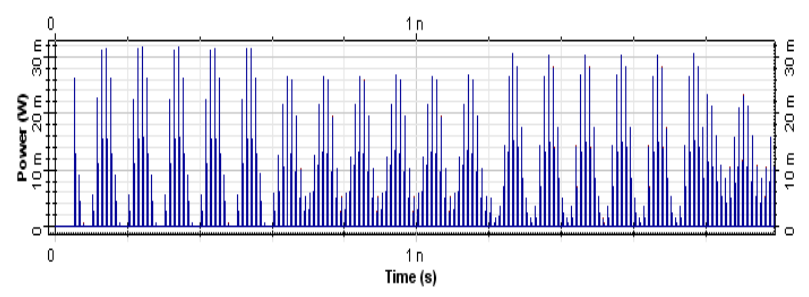

(b)
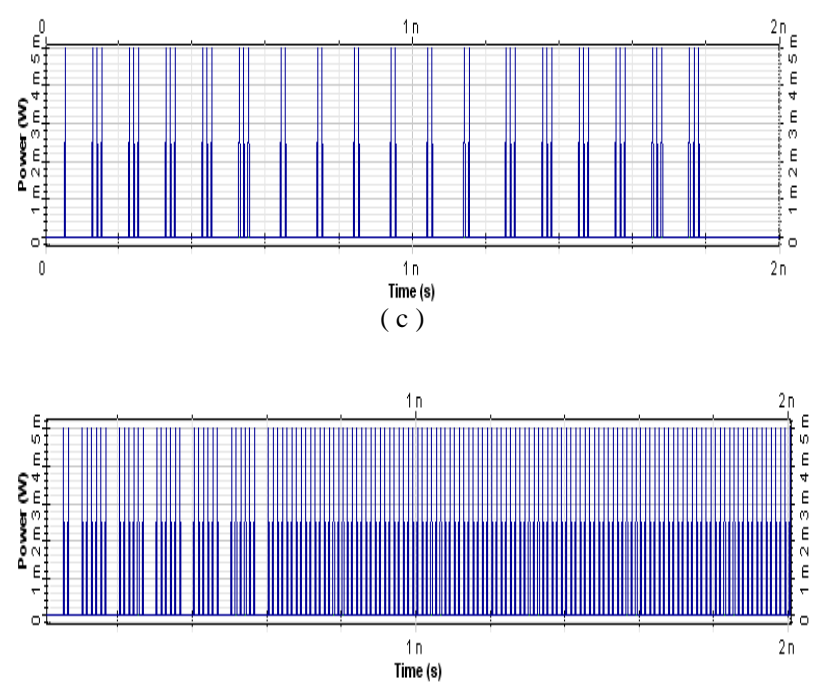

(d)

Figure 6: (a), A sample 64 QAM, 10 GHz RF signal, (b) Sampled optical signal, (c), Optical signal of MSB, (d) Optical signal of LSB.

Another limitation factor for PADC's performance is the effect of laser's jitter. If the laser pulses do not occur exactly when they should be, the signal will be sampled at the wrong time, and so the digitized output will differ from the signal at the assumed ideal jitter-free sampling time.

For investigation of the performance of the proposed digital radio over fibre system (DRoF), a sample 900 Mbit/s NRZ pseudo-random data is ASK modulated with $10 \mathrm{GHz}$ RF carrier and transported by the DRoF system. Fig. 7 shows the transmission system performance with the eye diagram of the received digital system. In this figure, the performance comparison of received signal by using digital and analogue systems has been shown over $35 \mathrm{~km}$ fibre length. In this system, the single mode fibre chromatic dispersion is assumed about $16 \mathrm{ps} /(\mathrm{nm} . \mathrm{km})$. By using a dispersioncompensated fibre, the dispersion has been reduced to 5 $\mathrm{ps} /(\mathrm{nm} . \mathrm{km})$.

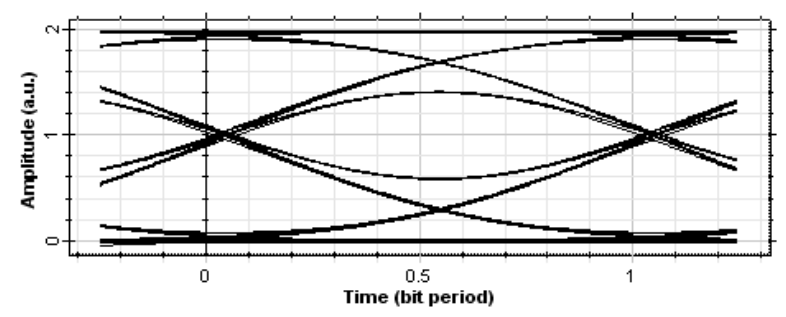

(a)

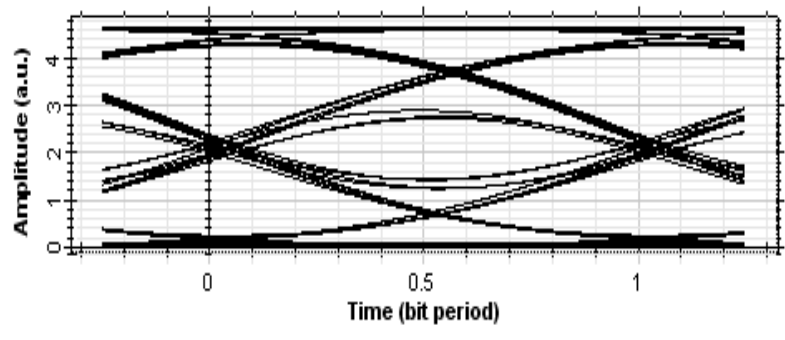

(b)

Figure 7. Eye diagram of $900 \mathrm{Mbit} / \mathrm{s}$ ASK modulated signal with 10 $\mathrm{GHz}$ carrier over $35 \mathrm{~km}$ length of single-mode fibre: (a) ARoF system, (b) DRoF system.

Fig. 8 shows detected $900 \mathrm{Mbit} / \mathrm{s}$ electrical signal of the (Analogue Radio over Fibre) ARoF and DRoF systems after transporting through $50 \mathrm{~km}$ single mode fibre, by using perfect chromatic dispersion compensation [1][9].

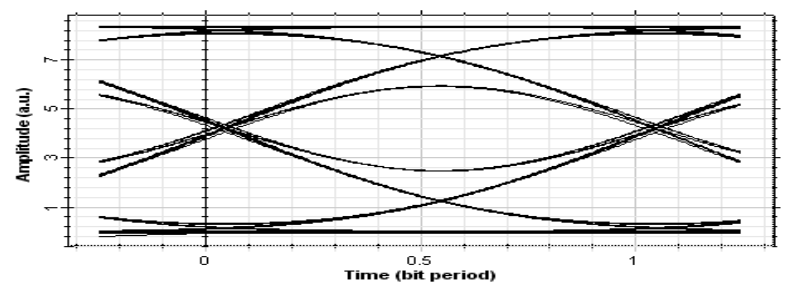

(a)

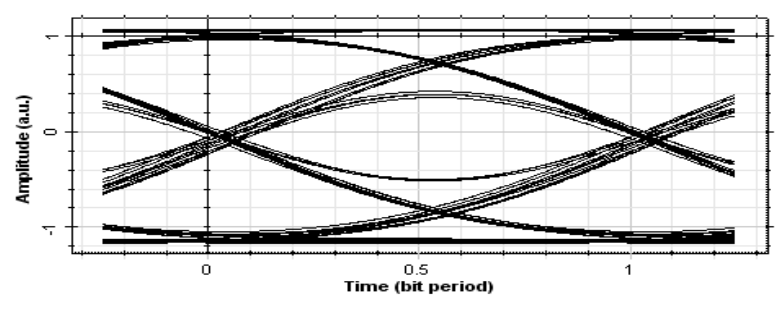

(b)

Figure 8: Eye diagram of $900 \mathrm{Mbit} / \mathrm{s}$ ASK modulated signal with 10 $\mathrm{GHz}$ carrier over $50 \mathrm{~km}$ length of single mode fibre: (a) ARoF system, (b) DRoF system.

\section{CONCLUSIONS}

In this paper, the proposed DRoF and conventional ARoF systems are simulated by Optiwave-Optisystem and Matlab simulation tools for super-broadband wireless signal transportation and distribution applications over $50 \mathrm{~km}$ single mode fibre. The performance of the novel DRoF link is investigated and compared with ARoF. In the DRoF system digitizing the analogue radio frequency (RF) signal and finally returning it back into analogue at destination is done by using our designed all photonic 8-bit ADC and DAC convertors. By using the proposed PADC, the $10 \mathrm{GHz}$, ASK modulated RF signal with $900 \mathrm{Mbit} / \mathrm{s}$ bandwidth is digitized with 80 Gigasample/s MLL pulse. The performance of photonic sampled and quantized ADC at 80 Gigasample/s is about six ENOB with $49.92 \mathrm{dBm}$ 
SNR. PADC's performance is affected by the laser's jitter, the nonlinearity of MZM, photonic amplifier and other photonic devices performance.

\section{REFERENCES}

[1] C. Lim, A. Nirmalathas, M. Bakaul, P. Gamage, K. L. Lee, Y. Yang, D. Novak, and R. Waterhouse, " Fiber-Wireless Networks and Subsystem Technologies," Journal of Lightwave Technology, vol. 28, no. 4, pp. 390-405, 2010.

[2] A. M. J. Koonen, M. Garcia Larrode, A. Ngoma, K. Wang, H. Yang, Y. Zheng, and E. Tangdiongga, " Prespectives of radio over fiber technologies”, IEEE Optical and Fiber Conference, pp 1-3, Feb. 2008.

[3] M. S. Rahman, J. H. Lee, Y. Park, and Ki-Doo Kim, " Radio over fiber as a cost effective technology for transmission of wimax signals", Journal of World Academy of Science, Enginnering and Technology, vol 56, pp. 424-428, 2009.

[4] H-C Ji, H. Kim, and Y. C. Chung, “ Full-duplex radio-over-fiber system using phase-modulated downlink and intensitymodulated uplink", IEEE Photonics Technology Letters, vol. 21, no. 1, pp. 9-11, Jan. 2009.

[5] G. C. Valley, "Photonic analog-to-digital converters," Journal of Lightwave Technology. vol. 15, no. 5, pp. 1955-1982, 2007.

[6] H. Al-Raweshidy and S. Komaki, Radio over Fiber Technology for Mobile Communication Networks, Artech House, 685 Canton Street, MA 02062, 2002. pp 136-138.

[7] C. H. Cox, III, Analog Optical Links, Cambridge University Press, Cambridge UK, 2004.

[8] G. L. Li. and P. K. L. Yu, "Optical intensity modulators for digital and analog applications,” Journal of Lightwave Technology. vol. 21, pp. 2010-2030, 2003.

[9] P. A. Gamage, A. Nirmalathas, C. Lim, D. Novak, and R. Waterhouse, "Design and analysis of digitized RF-Over-Fiber Links,” Journal of Lightwave Technology, vol. 27, no. 12, pp. 2052-2061, 2009.

[10] J. Kim, M. J. Park, M. H. Perrott, and F. Kartner, “ Photonic subsampling analog-to-digital conversion of microwave signals at $40-\mathrm{GHz}$ with higher than 7-ENOB resolution,” Optics Express, vol. 16, no. 21, pp. 16509-16515, 2008.

[11] R. H. Walden, “ Analog-to-digital converter survey and analysis," IEEE Journal of Selected Areas in Communications, vol. 17, no. 4, pp. 539-550, 1999. 